\title{
Piloting a trauma surveillance tool for primary healthcare emergency centres
}

\author{
I Govender, R Matzopoulos, P Makanga, J Corrigall
}

Objective. We aimed to pilot a trauma surveillance tool for use in a primary healthcare emergency centre to provide a risk profile of injury patterns in Elsies River, Cape Town.

Methods. Healthcare workers completed a one-page questionnaire capturing demographic and injury data from trauma patients presenting to the emergency unit of the Elsies River Community Health Centre over a period of 10 days.

Results. Trauma cases comprised about one-fifth of the total headcount during the study period. Most injuries took place before midnight. Approximately $47 \%$ of the trauma patients were suspected of being under the influence of alcohol with $87 \%$ of these cases caused by interpersonal violence; $28 \%$ were males between 19 and 35 years old, suspected of being under the influence of alcohol and presenting with injuries due to violence.

Conclusion. Injury surveillance at primary healthcare emergency centres provides an additional perspective on the injury burden compared with population-level mortality statistics, but the quality of data collection is limited by resource constraints. We recommend that the current trauma register be revised to separate trauma and medical headcounts and enable better resource planning at a facility and subdistrict level. Information gathered must be linked to health and safety interventions aimed at reducing the trauma burden within communities.

S Afr Med J 2012;102(5):303-306.

\section{Background}

The Western Cape Burden of Disease Study ${ }^{1}$ established that injuries due to interpersonal violence and road traffic crashes contribute to the 'quadruple burden of disease' reported in the province, i.e. poverty-related diseases, emerging chronic diseases, injuries and HIV/AIDS. ${ }^{1}$ Data for estimating the injury burden are usually drawn from vital registration or descriptive studies conducted at regional or central hospitals.-5 District or provincial-level data are useful for identifying broad health priorities and evaluating the impact of health interventions, but local-level data are equally important to identify locally relevant priority conditions, assist resource planning and inform targeted interventions. National or provincial statistics are also not always freely available, with delays in compilation, and are sometimes unreliable as a result of underreporting. ${ }^{6}$ Evaluations of the effectiveness of injury prevention and trauma systems should be based on data that are representative of the population during all phases of care. ${ }^{7}$ However, trauma surveillance at primary healthcare level in the Western Cape is currently non-existent.

Elsies River is located in the Tygerberg Health subdistrict of the Cape Metropole and is historically a predominantly 'coloured' area.

Provincial Government Western Cape and School of Public Health and Family Medicine, University of Cape Town Indira Govender, $\mathrm{MB} \mathrm{ChB}, \mathrm{MMed}, \mathrm{FCPHM}$

Medical Research Council, Parow, W Cape, and School of Public Health and Family Medicine, University of Cape Town

Richard Matzopoulos, BBusSci, MPhil (Epidemiology)

African Centre for Cities, University of Cape Town

Prestige Makanga, BSc Surveying and Goematics, MSc Eng Geomatics

Provincial Government Western Cape and School of Public Health and Family Medicine, University of Cape Town Joanne Corrigall, MB ChB, DMH, MMed, FCPHM
The after-hours trauma unit in the Elsies River Community Health Centre (CHC) was revamped in 2005 and serves over 43000 people in Tygerberg, the majority of whom are uninsured. ${ }^{8}$ Police statistics of the area show that contact crime has declined over the years but other crime requiring police action for detention, such as drugrelated crime and driving under the influence of alcohol or drugs, has increased. ${ }^{9}$ The Elsies River CHC Trauma Surveillance project was a complementary study to the Booza TV series community intervention project conducted in Elsies River in 2010. Booza TV is a series of six 30-minute documentaries that challenge prevailing drinking norms and promote the adoption of evidence-based methods to address alcohol abuse. The $\mathrm{CHC}$ was considered an important source of injury data to satisfy an objective of the intervention: conducting a community assessment regarding alcohol harms, prevention and use through a process of community consultation and analysis of available data sources. But inspection of the emergency centre (EC) register revealed that routine information was unsuitable for surveillance purposes, the primary concern being the aggregation of medical and trauma cases into a single emergency centre headcount. Distinguishing between trauma and non-trauma cases is of particular interest because of the strong association between alcohol and all types of injury. ${ }^{10}$ Other information regarding trauma cases was not routinely collected, such as demographic details, alcohol and drug use, the geographical location of the injury event and the type of injury (whether it related to violence, self-harm, a motor vehicle crash or other unintentional events preceding the injury).

The EC register functions as a patient organiser and was not designed for surveillance; hence a trauma surveillance tool for use in the 24-hour primary healthcare EC of Elsies River $\mathrm{CHC}$ was developed to gather this type of information. After receiving permission and support from the Tygerberg and Northern substructure director and the facility manager, the data collection tool was developed in consultation with senior EC nurses. The tool was a simple one-page form (Appendix A) with key elements based on the injury surveillance data capture tool developed by the South African Violence and Injury Surveillance for Violence Prevention Consortium. The information was limited to basic demographic data (age and gender); triage; cause, location of injury and period during daytime (before or after $1 \mathrm{pm}$ ) or night-time (before or after midnight) when it occurred; and whether 
the healthcare worker suspected the patient to be under the influence of alcohol or drugs. Our objectives were to pilot the tool and develop a risk profile for 23 different locales within Elsies River based on the information collected.

\section{Methods}

This cross-sectional descriptive study took place over 10 days in the EC of the Elsies River CHC from 25 November to 5 December 2010.

A feedback session was given to EC healthcare workers and the $\mathrm{CHC}$ reception staff to illustrate the objectives of the study, revise the protocol for data collection, and increase staff buy-in by presenting data that were collected during the first pilot that highlighted the potential uses of the information. Promotional and standard operating procedure posters were made for the reception office and the EC to raise awareness of the study and remind healthcare workers of the protocol.

Reception staff recorded the date, time and basic demographic details on the surveillance forms for $\mathrm{CHC}$ trauma patients and inserted these and triage forms into the patients' folders. In cases of uncertainty, reception staff filled in a surveillance form to err on the side of caution and patients were then triaged according to the South African Triage Scale. ${ }^{11}$ Healthcare workers completed the surveillance form for every trauma case noted, documenting the triage colour of the patient, the geographical location of the injury and the possible involvement of alcohol or other drugs. They placed a sticker in the EC register next to the recorded trauma cases to separate trauma from medical cases. At the end of the study period, the number of stickers in the register was used as a baseline to determine the completion rate of the trauma surveillance forms.

Informed consent was not requested as no additional information about the injury event was collected other than details routinely compiled by clinic staff. Case report forms were kept at a UCT safe storage facility. Electronic data were available only to members of the project team and were password protected. Anonymity and confidentiality were ensured by omitting personal identifiers on the case report form. The geospatial data used in the mapping process had resolution limited to the precinct boundaries, obviating the possibility of linking individual cases to specific times and locations. Ethical approval was granted by the University of Cape Town Research Ethics Committee (HREC Ref 508/2009) and the study was approved by the Provincial Health Research Committee.

Univariate analysis was undertaken in Microsoft Excel 2007. The results were georeferenced and spatially analysed using ArcGIS 10.

\section{Results}

During the study period there were a total of 1252 entries in the EC register with $8.5 \%$ (107) labelled as 'called and no response', i.e. the patient was triaged but left the facility before being seen by a healthcare worker. On average $97 \%$ of the trauma cases in the register identified by a sticker had a completed surveillance form, but this completion rate differed widely between day and night shift (Table I).

Table 1. Summary of EC register totals

\begin{tabular}{lll}
\hline Day shift & Register total & 459 \\
& No. of stickers in the register & 76 \\
Night shift & No. of trauma surveillance forms & 95 \\
& Register total & 793 \\
& No. of stickers in the register & 171 \\
& No. of trauma surveillance forms & 145
\end{tabular}

At the end of the study period 257 surveillance forms were returned categorised as follows: 221 trauma, 5 medical and 31 unknown; 20 of the unclassified cases were interpreted as trauma cases based on responses to 'Circumstance/Cause of injury' item on the surveillance form. An unclassified case that had a cause of trauma recorded, was counted as trauma.

Out of 241 trauma cases that presented to the CHC, $16 \%$ of injuries occurred during the day before $1 \mathrm{pm}, 23 \%$ after $1 \mathrm{pm}, 39 \%$ at night before midnight and $19 \%$ after midnight. Of the triage colours, $90 \%$ were yellow (presented with controlled haemorrhage; abdominal pain; closed fracture; dislocation of finger or toe; burns other than those classified as orange or moderate pain); $4 \%$ were orange (uncontrolled haemorrhage; other joint dislocations; compound fractures; $>20 \%$ burns or severe pain) and $5 \%$ were unclassified. Most male and female trauma cases were between the ages of 19 and 35 years (Fig. 1).

Of trauma cases that reported to Elsies River EC, $70 \%$ were caused by violence (Fig. 2).

According to the clinical judgement of EC healthcare workers, $47 \%$ of trauma patients were suspected of being under the influence of alcohol (Fig. 3).

Of the 113 trauma cases suspected of being under the influence of alcohol, $87 \%$ of injuries were due to violence, $4 \%$ due to self-harm, $2 \%$ were traffic related, $4 \%$ classified as other and $3 \%$ unknown.

Most incidents took place in Elsies River (53\%) and Bishop Lavis (13\%), $28 \%$ occurred in other areas and $6 \%$ of locations were

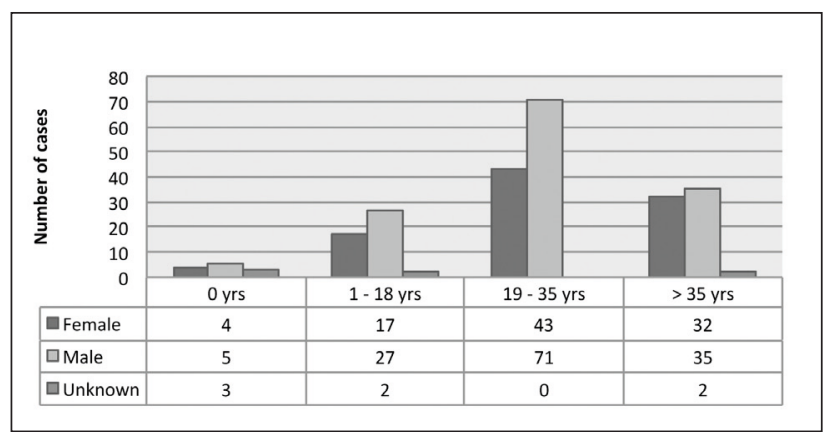

Fig. 1. Age and gender of trauma cases $(\mathrm{N}=241)$.

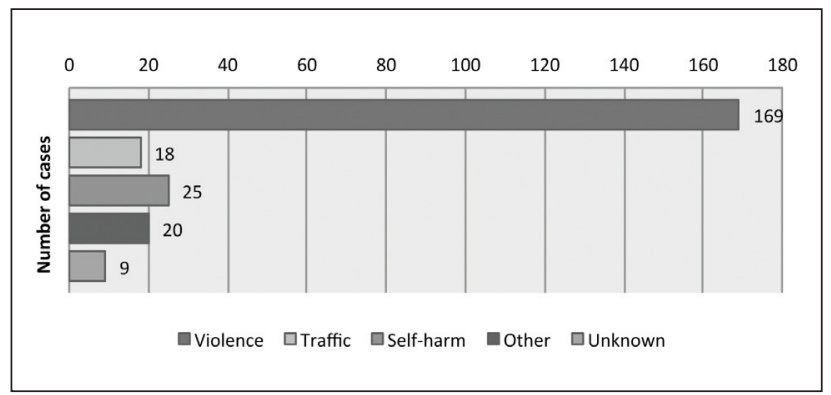

Fig. 2. Causes of trauma ( $\mathrm{N}=241)$.

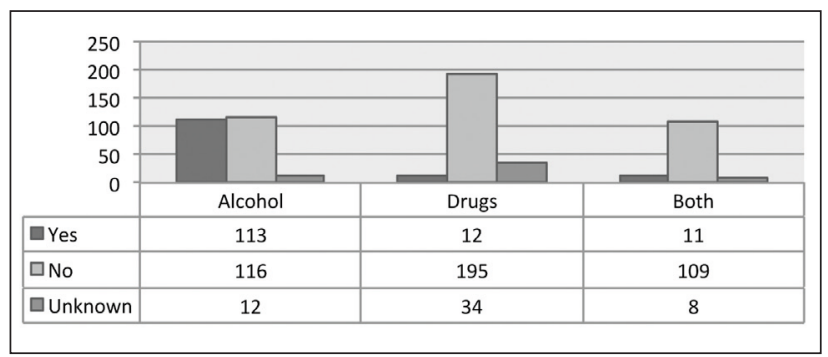

Fig. 3. Trauma cases suspected of using alcohol and/or drugs. 
unknown. Of the 128 cases that occurred in Elsies River 118 (53\%) were linked to specific locales with five areas (Uitsig, Leonsdale, Elsies River, Clarke Estate and Connaught) reporting the highest numbers of trauma cases.

\section{Discussion and recommendations}

Use of a simple surveillance tool provided a snap-shot of the trauma caseload at a 24-hour EC in a primary healthcare facility that serves an area of low socio-economic status. The disaggregation of EC headcounts into trauma and non-trauma allows for better resource planning within the facility, and provides local data to inform resource distribution for health prevention and promotion efforts within the community. The focused caseload and catchment area of the $\mathrm{CHC}$ allowed for finer spatial resolution of areas at risk than might be achieved via large secondary or tertiary facilities. ${ }^{5}$ This is relevant for providing targeted interventions in high-risk communities as sharing injury and emergency data with other local authorities is effective in bringing about violence reduction. ${ }^{12}$

Cases of major trauma such as motor vehicle crashes or severe gunshot wounds are usually taken directly to regional or tertiary emergency centres by emergency medical services. Therefore community trauma profiles collected at these centres, or based on data extrapolated from mortality surveillance, are likely to conceal underlying social pathologies, such as alcohol misuse and domestic violence. This primary healthcare study showed that most traumatic incidents took place before midnight, were violence related and resulted in injuries triaged as yellow. Violence was also the main cause of trauma in patients suspected of being under the influence of alcohol, in keeping with other studies. ${ }^{1}$ It has been argued that risk factors such as alcohol use, youth, male gender and socioeconomic status that are predictive of trauma recidivism (referring to patients who require medical care for repeated injury episodes) are also predictive of incarceration recidivism. ${ }^{14}$ The majority of trauma patients presenting to the Elsies River CHC EC were males between the ages of 19 and 35 years, and further research or more detailed surveillance may be beneficial in identifying repeat presentations for specific prevention strategies.

This study had few limitations, primarily integrating data collection with the work flow in the EC so that healthcare workers had an additional administrative burden that probably negatively affected the quality of data collection during busy periods. Along with missing data and incomplete forms, there was a discrepancy in the number of trauma cases marked in the EC register and the number of trauma surveillance forms collected. The difference between day and night shift data collection may have produced an inaccurate estimate of the proportion of trauma cases out of the total EC headcount and the time of occurrence of traumatic incidents. Another concern was staff changeovers between day and night shifts, as direct protocol training and feedback were only provided to healthcare workers during the day, who were then expected to hand over data collection to those working at night.

Data collection took place over a weekend at the end of the month when most employed individuals are paid. It is likely that the proportion of trauma was exaggerated, since health facilities report abnormally high levels of violence and injuries over this period, probably linked to alcohol purchase.
Another limitation was the unvalidated questions on suspected alcohol and drug use. Even though caregiver assessment may be sufficient to establish whether alcohol is involved in trauma, these questions relied on healthcare workers' clinical judgement without objective measurements and are likely to have produced inaccurate estimates of the proportion of trauma cases under the influence of alcohol or drugs. ${ }^{15}$ Given that $47 \%$ of trauma patients were suspected of being under the influence of alcohol, it might be worth investing in rapid alcohol tests, such as breathalysers, for ECs to facilitate appropriate referral or rehabilitation. Further research is needed to investigate the sensitivity and feasibility of caregiver assessment versus a breath alcometer, for alcohol only, and physiological measures for drugs and alcohol in the form of urinalysis.

Our findings suggest that planning within ECs, and health services in general, will benefit from amending the current $\mathrm{EC}$ register so that trauma and medical headcounts are tallied separately. If this study is replicated at other health facilities, the information gathered should be linked to health and safety interventions aimed at reducing the trauma burden within communities. Further, following health and safety interventions in the Elsies River community, a repetition of this study at the $\mathrm{CHC}$ will enable measurement of changes in risk over time and assessment of the effectiveness of prevention efforts.

Acknowledgements. We thank the Open Society Foundation - South Africa for funding, Sisters Patricia Macpherson and Marilyn Patterson, the reception staff and emergency centre healthcare workers at Elsies River Community Health Centre for their co-operation and work piloting the surveillance tool.

\section{References}

1. Bradshaw D, Groenewald P, Laubscher R, et al. Initial Burden of Disease Estimates for South Africa 2000. Cape Town: South African Medical Research Council, 2003.

Hanewinckel R, Jongman HP, Wallis LA, Mulligan TM. Emergency medicine in Paarl, South Africa: cross-sectional descriptive study. Int J Emerg Med 2010;3:143-150.

Naledi T, Househam KC, Groenewald P, Bradshaw D, Myers JE Improving data to reduce the burden of disease: lessons from the Western Cape. S Afr Med J 2009;99(9):641-642.

. Norman R, Matzopoulos R, Groenewald P, Bradshaw D. The high burden of injuries in South Africa. Norman R, Matzopoulos R, Groenewald P, Bradl

Schuurman N, Cinnamon J, Matzopoulos R, Fawcett V, Nicol A, Hameed SM. Collecting injury Schuurman N, Cinnamon J, Matzopoulos R, Fawcett V, Nicol A, Hameed SM. Collecting injury
surveillance data in low- and middle-income countries: The Cape Town Trauma Registry pilot. Global surveillance data in low- and middle-income countries: The Cape Town Trauma Registry pilot. Global
Public Health. 2010. DOI:10.1080/17441692.2010.516268.

6. Mock C, Joshipura M, Quansah R, Arreola-Risa C. Advancing injury prevention and trauma care in North America and globally. Surg Clin N Am 2006;87:1-19.

7. Mullins RJ, Clay N. Population-based research assessing the effectiveness of trauma systems. The Journal of Trauma: Injury, Infection and Critical Care 2009;47(3):59-56.

8. Cape Gateway (2006) http://www.capegateway.gov.za/eng/pubs/news/2005/nov/12241 (accessed 29 May 2011)

Crime Information Management - South African Police Service (2010) http://www.saps.gov.za/ statistics/reports/crimestats/2010/provinces/w_cape/pdf/elsiesriver.pdf (accessed 29 May 2011).

10. Ye Y, Cherpitel CJ. 2009. Risk of injury associated with alcohol and alcohol-related injury. In: Cherpite CJ Borges $G$ Giesbrecht $N$, et al, eds. Alcohol and Injuries. Geneva: World Health Organization, 2009:3-14.

11. South African Triage Group. South African Triage Scale Hospital Provider Manual. Edition 2. Cape Town: South African Triage Group, 2008. Warburton AL, Shepherd JP. Development, utilisation, and importance of accident and.
department derived assault data in violence management. Emerg Med J 2004;21:473-477.

13. Borges G, Macdonald S, Cherpital CJ, Orozco R, Peden M. Variation in alcohol-related injury by type and cause of injury. In: Cherpitel CJ, Borges, G, Giesbrecht, N, et al., eds. Alcohol and Injuries. Geneva: World Health Organization, 2009:15-26.

14. Kaufmann CR, Branas CC, Brawley ML. A population-based study of trauma recidivism. The Journal of Trauma: Injury, Infection and Critical Care 1998;45(2):325-332.

15. Marais S. WHO collaborative study on alcohol and injuries: South Africa. South African Medical Research Council Technical Report. Cape Town: South African Medical Research Council, 2002.

Accepted 13 November 2011. 
Appendix A

\section{ELSIES RIVER COMMUNITY HEALTH CENTRE - TRAUMA RECORD}

\section{Date: $\square$ Oct 2010}

HREC REF: 508/2009

1.

\begin{tabular}{|l|l|}
\hline Day shift & \\
\hline
\end{tabular}

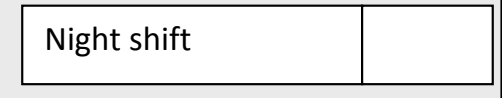

2. Triage colour?
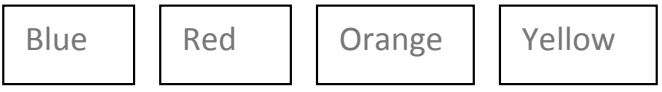

Green
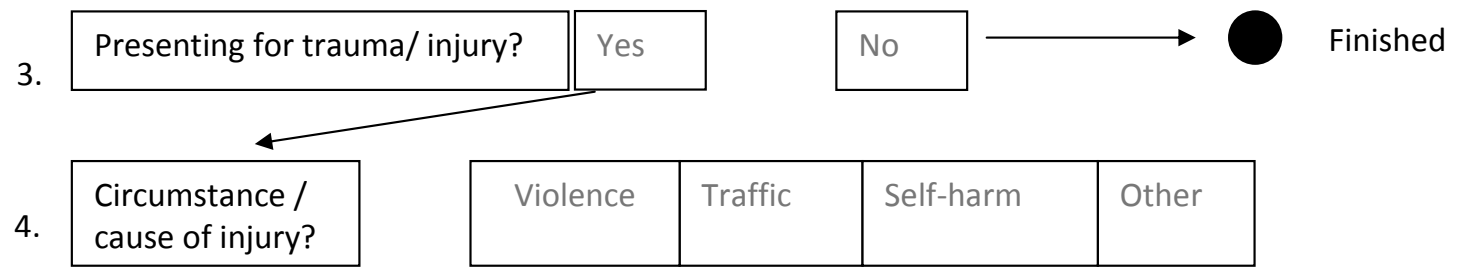

5.

\begin{tabular}{|l|l|l|}
\hline Suspected alcohol? & Yes & No \\
\hline
\end{tabular}

6. Suspected drugs?

\begin{tabular}{|l|l|}
\hline Yes & No \\
\hline
\end{tabular}

7. When did it happen?

Daytime

Nighttime

\begin{tabular}{|l|l|}
\hline Before $1 \mathrm{pm}$ & After $1 \mathrm{pm}$ \\
\hline Before midnight & After midnight \\
\hline
\end{tabular}

8. Age in years?
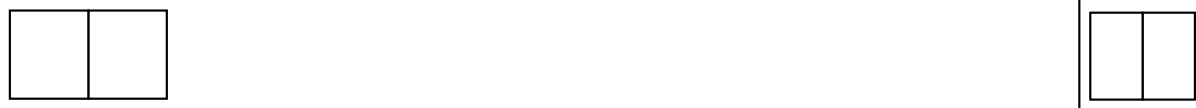

Gender

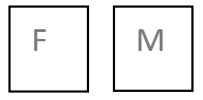

10. Where was the person injured?

Elsies River

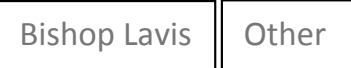

Finished

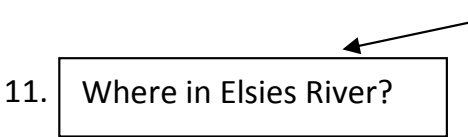

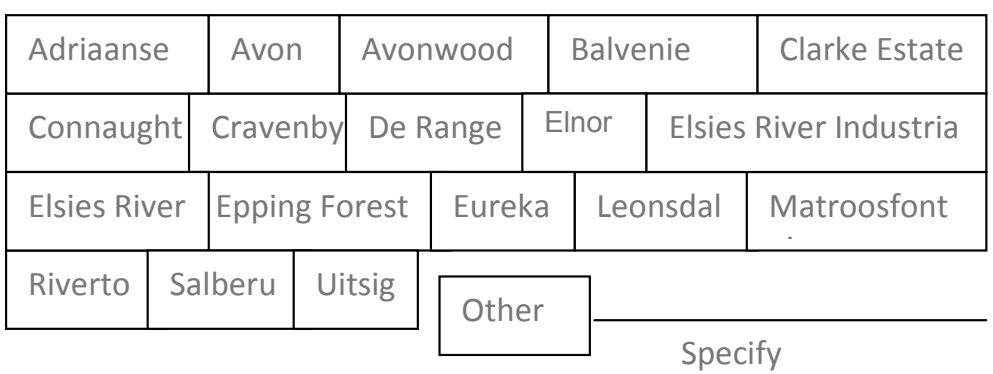

12. 